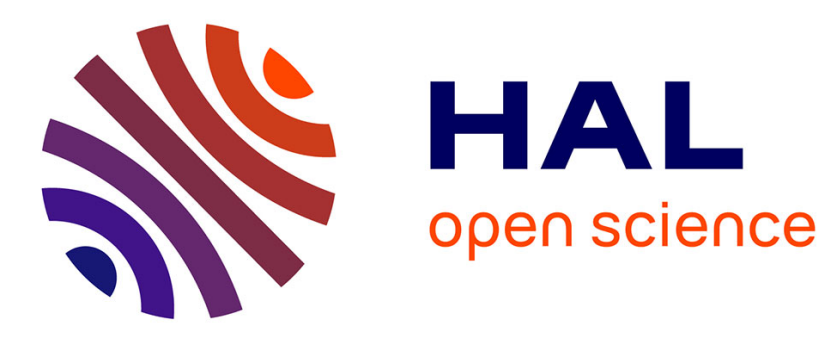

\title{
Espaces homosexuels dans la ville
}

Arnaud Alessandrin, Yves Raibaud

\section{To cite this version:}

Arnaud Alessandrin, Yves Raibaud. Espaces homosexuels dans la ville. Hermes - Journal of Language and Communication Studies, 2014, Sexualités (69), pp.152-154. 10.3917/herm.069.0152 . hal01059352

\section{HAL Id: hal-01059352 \\ https://hal.science/hal-01059352}

Submitted on 29 Aug 2014

HAL is a multi-disciplinary open access archive for the deposit and dissemination of scientific research documents, whether they are published or not. The documents may come from teaching and research institutions in France or abroad, or from public or private research centers.
L'archive ouverte pluridisciplinaire HAL, est destinée au dépôt et à la diffusion de documents scientifiques de niveau recherche, publiés ou non, émanant des établissements d'enseignement et de recherche français ou étrangers, des laboratoires publics ou privés. 


\title{
Espaces homosexuels dans la ville
}

\author{
Revue Hermès n69 août 2014, CNRS éditions, Paris, p.152-154 \\ Arnaud Alessandrin, centre Émile Durkheim, UMR 5116 \\ Yves Raibaud, Adess Cnrs, université Bordeaux Montaigne
}

Grindr est une application pour téléphones intelligents (smartphones) destinée aux rencontres homosexuelles par géolocalisation, qui compte aujourd'hui trois cent mille utilisateurs en France ${ }^{1}$. Les rencontres avec Grindr brouillent les interprétations classiques des géographies homosexuelles, centrées sur les lieux de drague, commerces et quartiers spécifiques, toutes marquées par les frontières des normes hétérosexistes. Grindr peut être envisagé comme un géoindicateur des rencontres homosexuelles et comme un instrument de relocalisation de ces rencontres, par un repérage des partenaires potentiels sur une carte interactive du territoire où l'on se situe, avec accès au profil et aux préférences.

Dans son «épistémologie du placard» (2008), Eve Kosofsky Sedwick rappelle que le placard ne divise pas l'espace entre connaissances du dedans et peurs du dehors, mais que l'homo-érotisme, l'homophobie et l'hétérosexualité ont tissé des liens particuliers (dans des gestes d'assimilation ou de transgression). Or, avec Grindr, l'appréhension de la ville par les homosexuels $^{2}$ ne s'articule plus autour d'une logique de séparation d'espaces érotiques ou anxiogènes, conditionnant un usage spécifique de la ville, mais selon une logique de frottements et de multiplicité dans l'urbain : le sentiment d'insécurité se dilue dans l'espace. Dans ce contexte, les homosexuel-le-s expérimentent des usages de l'urbain à la fois prescrits et fortuits, à l'image d'une homosexualité qui n'est plus caractérisée par une opposition forte entre le privé et le public, mais par la porosité de ces mêmes espaces.

\section{La ville « grinderisée »}

L'application rebat les cartes de la visibilité « gay » en atomisant les espaces de rencontres, les transformant au hasard du lieu et des connexions. Avec Grindr, la cartographie des rencontres ne repose pas sur le principe de lieux matériels mais des flux. La seule question qui se pose est où l'on est: il s'agit non seulement d'être prêt, mais aussi d'être rapidement présent. Alors que l'histoire des homosexualités est aussi l'histoire de ses lieux de rencontres, Grindr n'a pas besoin de lieu. Il a besoin d'interactions, de déplacements : on ne se déplace plus dans un lieu de rencontres, mais avec un outil de rencontres. Cela produit un émiettement des lieux de rencontres et donc, probablement, une reconfiguration des espaces communautaires. L'exemple d'une ville comme Bordeaux est assez probant. Les quais et les terrasses de Mériadeck ont été rénovés. La lumière des lieux et les espaces de promenade en font des espaces familiaux et non des espaces de rencontres. Quant au quartier du Lac et aux aires d'autoroute périphériques, ils ont tout autant perdu en réputation. Grindr, et avec lui l'ensemble des outils numériques à destination de la communauté homosexuelle gay, dé-territorialise l'espace des rencontres, ou alors le multiplie. En anglais, grindr signifie «moulin», mais aussi «broyeur». S’il mélange, fluidifie, il détruit tout autant, ou recompose les espaces de rencontres. La rue, les bars, les boîtes et les backrooms font toujours office d'espaces de rencontres et de socialisation, mais leur centralité est aujourd'hui « en délibéré ».

Ajoutons que cette observation pose également la question de l'inscription de l'homosexualité dans la ville : assiste-t-on à une dilution, une assimilation, une invisibilisation?

\footnotetext{
${ }^{1}$ En 2012, on comptait 260000 inscrits en France, dont 200000 à Paris. Aujourd'hui, des applications comme Scruff ou Hornet viennent concurrencer Grindr en proposant une offre plus étendue de services. Des applications à destination des publics hétérosexuels ou lesbiens sont aussi disponibles, mais paraissent moins utilisées. En ce sens, le poids des rencontres sexuelles chez les gays, notamment à Paris, semble conforté par l'utilisation de ces applications.

${ }^{2}$ Grindr n'étant utilisé que par des gays, nous employons ici le masculin.
} 
Grindr en est-il le révélateur? Il faut admettre les difficultés qu’il y a à cartographier les espaces invisibles des rencontres anonymes, celles justement auxquelles participent les utilisateurs de plus en plus nombreux de l'application. Cette dilution du sexuel dans l'urbain (Toulze, 2013) réinterroge aussi la notion d'homophobie dans un contexte où l'augmentation tendancielle des droits des minorités n’inaugure en rien l'absence du sentiment homophobe.

\section{Grindr comme géo-indicateur et outil de relocalisation}

Grindr est une interface entre espace et cyberespace. Dans notre esprit, le cyberespace n'est pas considéré comme un espace irréel, ni même comme un espace hyperréel qui performerait la « réalité » des sociétés humaines, mais comme un lieu d'hébergement d'actants ou opérateurs (Lussault, 2014, p.51), fonctionnant comme des quasi-personnages autour desquels s'organisent des mondes sociaux. Grindr est un opérateur qui interagit entre différents niveaux d'espaces, à moins qu'il ne s'agisse que du même espace, successivement et/ou simultanément perçu, représenté et vécu par des «individus spatiaux» (Raibaud, 2011, p.49) qui utilisent l'application. Une certaine géographie représentait par des cartes un monde dans lequel les rapports de domination économiques et sociaux, mais aussi de «race» et de sexe, sont profondément ancrés, mais tout aussi profondément occultés. Il y a encore peu de temps la géographie des sexualités n'échappait pas à cette règle : seules les sexualités officielles y étaient visibles, souvent dans les travaux des démographes (géographie de la famille, de la fécondité), sauf lorsque la sexualité apparaissait comme un facteur marquant et fortement hiérarchisé des sociétés humaines, par exemple entre le Nord et le Sud (cartographie de la polygamie, de l'excision, du divorce). En explorant les lieux de rencontre des «minorités sexuelles », on dévoile un espace des sexualités jusqu'alors inconnu : lieux de rencontres dans l'espace public, parcs, aires de stationnement, plages, bars et commerces, fêtes privées, mais aussi lieux où l'on se fait voler, insulter, tabasser (Cattan et Leroy, 2013).

L'espace officiel des sexualités étant l'espace des sexualités officielles, Grindr agit donc comme un géo-indicateur d'autres sexualités. Il suffirait de connecter le principe des cartographies 2.0 à Grindr pour voir apparaittre instantanément une géographie gay (voire plus largement lesbienne, gay, bi et trans) des usages de la ville. Une approche de cartographie participative, avec l'aide des utilisateurs, pourrait représenter les flux et les stationnements par espaces/temps, d'articuler les espaces érotiques/espaces anxiogènes permettant de mieux comprendre les peurs et les désirs des "minorités sexuelles» dans la ville, tels qu'ils ont été approchés dans Géographie des homophobies (Alessandrin et Raibaud, 2013). La rencontre sexuelle immédiate favorisée par Grindr est une possibilité de (ré)investir des espaces considérés comme hostiles du fait de leurs supposées normes sexuelles, mais aussi sociales ou « raciales ». Le fait que les usagers puissent être à chaque instant informés des lieux de rencontres, fêtes ou lieux ressources peut favoriser l'empowerment des populations les plus discriminées (on pense notamment aux personnes trans). La rencontre immédiate par géolocalisation n'exclut pas, au contraire, l'agrégation à des groupes, pour lesquels l'égalité d'accès à l'espace représente une des conditions premières de l'exercice d'une citoyenneté pleine et entière.

Alessandrin, A. et Raibaud, Y. (dir.), Géographie des homophobies, Paris, Armand Colin, 2013.

Cattan, N. et Leroy, S., avec la coll. de MARIn, C. (cartographie), Atlas mondial des sexualités. Libertés, plaisirs et interdits, Paris, Autrement, coll. « Atlas/Monde », 2013.

KOSOFSKY SEDWICK, E., Épistémologie du placard, Paris, éditions Amsterdam, 2008.

Lussault, M., Actant, in Dictionnaire de la Géographie et de l'Espace des Sociétés, Paris, Belin, p. 51-52, 2014

Miroir/Miroirs, n 1, dossier « Grindr mon amour ? », 2013.

Raibaud, Y., Géographie socioculturelle, Paris, L’Harmattan, coll. « Logiques sociales », 2011.

Toulze, M., « Regard sur la ville ou l'hypertrophie numérique », Miroir/Miroirs, n 1, dossier « Grindr mon amour », 2013. 